\title{
On the Faddeev-Jackiw symplectic framework for topologically massive gravity
}

\author{
Alberto Escalante ${ }^{1, a}$, Omar Rodríguez-Tzompantzi ${ }^{2, b}$ \\ ${ }^{1}$ Instituto de Física, Benemérita Universidad Autónoma de Puebla, Apartado postal J-48, 72570 Puebla Pue., México \\ ${ }^{2}$ Facultad de Ciencias Físico Matemáticas, Benemérita Universidad Autónoma de Puebla, Apartado postal 1152, 72001 Puebla Pue., México
}

Received: 30 March 2016 / Accepted: 9 October 2016/ Published online: 25 October 2016

(C) The Author(s) 2016. This article is published with open access at Springerlink.com

\begin{abstract}
The dynamical structure of topologically massive gravity in the context of the Faddeev-Jackiw symplectic approach is studied. It is shown that this method allows us to avoid some ambiguities arising in the study of the gauge structure via the Dirac formalism. In particular, the complete set of constraints and the generators of the gauge symmetry of the theory are obtained straightforwardly via the zero modes of the symplectic matrix. In order to obtain the generalized Faddeev-Jackiw brackets and calculate the local physical degrees of freedom of this model, an appropriate gauge-fixing procedure is introduced. Finally, the similarities and relative advantages between the Faddeev-Jackiw method and Dirac's formalism are briefly discussed.
\end{abstract}

\section{Introduction}

Theories of interacting spin-2 fields, such as massive gravity, have been considerably studied in the literature, with particular focus on their symmetries and physical degrees of freedom [1-12]. The construction of a unitary and renormalizable theory of gravity with propagating degrees of freedom has been a long-sought goal toward our understanding of gravitation. In this context, it is well known that the Fierz-Pauli theory provides a consistent description of the linear fluctuations of a massive graviton on a flat space-time [13]. The Boulware-Deser (BD) ghost mode was exactly found for the Fierz-Pauli theory taken at the nonlinear level $[14,15]$, which violates the unitarity of the theory (a condition of consistency in quantum gravity). For this reason, the construction of an action for nonlinear massive gravity must ensure the absence of any ghost-like unphysical degrees of freedom, thereby rendering a stable and consistent theory. Strictly speaking, the theory must possess the necessary dynamical constraints for removing the ghost degrees of freedom, Nonetheless, it

\footnotetext{
a e-mail: aescalan@ifuap.buap.mx

be-mail: omar.tz2701@gmail.com
}

would be interesting if one could systematically obtain the constraints that eliminate such ghost fields. This approach would also be helpful to understand the gauge structure and the physical content of this kind of theories. In this work, we are interested in the study of the three-dimensional version of a massive gravity theory.

It is well known that the key ingredient for understanding the physical content of a gauge dynamic system lies in the identification of the physical degrees of freedom along with observable quantities and symmetries. Therefore, in a gauge theory it is essential to make the distinction between gauge-invariant (gauge-dependent) quantities, which do (do not) correspond to observable quantities [16,17], though the former are not necessarily present at the quantum level. The task of identifying the symmetries and observable quantities in a physical theory is, in general, non-trivial, specially in gauge theories with general covariance, such as general relativity. Nevertheless, there are two approaches to obtain in a systematic way the symmetries and conserved quantities of a particular physical theory: Dirac's formalism [18] and the Faddeev-Jackiw [FJ] method [19]. In the former approach, it is necessary to classify all constraints into first- and secondclass ones. As a consequence, the physical degrees of freedom can be exactly counted, and a generator of the gauge symmetry can be constructed as a suitable combination of the first-class constraints in order to identify the physical observables [20]. Furthermore, the brackets to quantize a gauge system (Dirac's brackets) can be obtained by getting rid of the second-class constraints [21,22]. On the other hand, the F-J method provides a symplectic approach for constrained systems based on a first-order Lagrangian. The basic feature of this approach is that it is not necessary to classify the constraints into first- and second-class ones. Still, several essential elements of a physical theory, such as the degrees of freedom, the gauge symmetry, and the quantization brackets (generalized F-J brackets) can also be obtained (see [23, 24, 27-29] for a review). In this framework, the non- 
null F-J brackets emerge from the symplectic matrix. For a gauge system, this matrix remains singular unless a gaugefixing procedure is introduced. In addition, the generators of the gauge symmetries are given in terms of the zero modes of this symplectic matrix. In this respect, the F-J symplectic method provides a straightforward effective tool to deal with gauge theories because it is algebraically simpler than Dirac's formalism. In particular, if secondary, tertiary, or higher order constraints are present.

Quite recently, the F-J symplectic method has proved useful in the study of many physical theories, for instance in the construction of Maxwell-inspired $S U$ (3)-like and $S U(3) \otimes S U(2) \otimes U(1)$ non-Abelian theories [30], as well as noncommutative gauge theories [31]. Furthermore, this approach not only has been useful to study non-Abelian systems [32], hidden symmetries [33], and self-dual fields [34], but also to quantize massive non-Abelian Yang-Mills fields [35] fields and to study the extended Horava-Lifshitz gravity [36]. For other work on the F-J symplectic approach we refer the interested reader to Refs. [37-39].

The purpose of the present work is to present a detailed study of three-dimensional topologically massive gravity (TMG) in a completely different context to that presented in $[40,42,43,47]$. It is well known that the canonical analysis of TMG is a large and tedious task since there are present secondary, tertiary and quartic constraints with a complicated algebra $[43,47]$. On the other hand, it is possible that if one step of the Dirac formalism is either incorrectly applied or omitted [48,50], the results could be incorrect $[42,47]$. In this respect, we will apply the F-J symplectic approach to systematically obtain the constraints necessary to remove the unphysical degree of freedom of the theory, the gauge symmetries, and the fundamental F-J brackets by introducing an appropriate gauge-fixing procedure. Moreover, the similarities and relative advantages between this procedure and Dirac formalism will be discussed. It also will be shown that the physical degrees of freedom, the gauge symmetries and the brackets to quantize agree with those found via the Dirac method in $[42,43,47]$.

The rest of the paper has been organized as follows. In Sect. 2, we show that the F-J symplectic method applied to TMG leads to an alternative way for identifying the dynamical constraints. In Sect. 3, the gauge transformations are obtained using the zero modes of the symplectic two-form matrix. In Sect. 4, we show that both the fundamental F-J brackets and the physical degrees of freedom are obtained by introducing a gauge-fixing procedure. In Sect. 5, we present a summary and the conclusions.

\section{Faddeev-Jackiw symplectic approach to TMG}

The action for TMG can be written as $[42,43,47]$

$$
\begin{aligned}
S[A, e, \lambda]=\int_{\mathcal{M}} & {\left[2 \theta e^{i} \wedge F[A]_{i}+\lambda^{i} \wedge T_{i}+\frac{\theta}{\mu} A^{i}\right.} \\
& \left.\wedge\left(\mathrm{d} A_{i}+\frac{1}{3} f_{i j k} A^{j} \wedge A^{k}\right)\right],
\end{aligned}
$$

where $\mu$ is the Chern-Simons parameter, $\theta=1 / 16 G$, and $A^{i}=A_{\mu}{ }^{i} \mathrm{~d} x^{\mu}$ is a connection one-form valued on the adjoint representation of the Lie group $S O(2,1)$, which admits an invariant totally antisymmetric tensor $f_{i j k}$. Furthermore $e^{i}=$ $e_{\mu}^{i} \mathrm{~d} x^{\mu}$ is a triad one-form that represents the gravitational field and $F^{i}$ is the curvature two-form of the connection $A^{i}$, i.e., $F_{i} \equiv \mathrm{d} A_{i}+\frac{1}{2} f_{i j k} A^{j} \wedge A^{k}$. Finally $\lambda^{i}$ is a Lagrange multiplier one-form that ensures that the torsion vanishes $T_{i} \equiv d e_{i}+f_{i j k} A^{j} \wedge e^{k}=0$.

The equations of motion that arise from the variation of the action (1) with respect to the dynamical variables $e_{\alpha}{ }^{i}$, $A_{\alpha}{ }^{i}$, and $\lambda_{\alpha}{ }^{i}$ are given, in addition to some total derivative terms, by

$$
\begin{aligned}
& (\delta e)^{\alpha i}=\epsilon^{\alpha \nu \rho}\left(2 \theta F_{\nu \rho}{ }^{i}+D_{\nu} \lambda_{\rho}{ }^{i}\right)=0 \\
& (\delta A)^{\alpha i}=\epsilon^{\alpha \nu \rho}\left(2 \theta T_{\nu \rho}{ }^{i}+f_{j k}^{i} \lambda_{\nu}{ }^{j} e_{\rho}{ }^{k}+2 \theta \mu^{-1} F_{\nu \rho}{ }^{i}\right)=0 \\
& (\delta \lambda)^{\alpha i}=\epsilon^{\alpha \nu \rho} T_{\nu \rho}{ }^{i}=0 .
\end{aligned}
$$

From the second and third equation in (2), the Lagrange multiplier $\lambda_{\mu}{ }^{i}$ can be solved in terms of the Schouten tensor of the manifold $\mathcal{M}$

$\lambda_{\mu}{ }^{i}=2 \theta \mu^{-1} S_{\mu \nu} e^{i \nu}, \quad$ with

$S_{\mu \nu}=(R i c)_{\mu \nu}-\frac{1}{4} g_{\mu \nu} R$.

The manifold is endowed with a space-time metric, $g_{\mu \nu}=$ $e_{\mu}{ }^{i} e_{\nu}^{j} \eta_{i j}$. Furthermore, since the torsion vanishes, the spinconnection $A_{\mu}{ }^{i}$ is a function of the dreibein $e_{\mu}{ }^{i}$

$A_{\mu}^{i j}=-e^{\nu j} \partial_{\mu} e_{\nu}^{i}+\Gamma_{\alpha \mu}^{\beta} e_{\beta}^{i} e^{\alpha j}$,

where $\Gamma_{\alpha \mu}^{\beta}$ is the Christoffel symbol. Inserting Eq. (3) into the first equation of (2), one gets the usual field equation of TMG $[2-4,40]$ in the second-order formalism

$G_{\mu \nu}+\frac{1}{\mu} C_{\mu \nu}=0$,

where $G_{\mu \nu} \equiv R_{\mu \nu}-\frac{1}{2} g_{\mu \nu} R$ is the Einstein tensor, and $C_{\mu \nu} \equiv \epsilon_{\mu}{ }^{\alpha \beta} \nabla_{\alpha} S_{\beta \nu}$ is essentially the (symmetric traceless ) Cotton tensor obtained from varying the gravitational ChernSimons term with respect to the metric, with $\nabla=\partial+\Gamma$ the covariant derivative for the Christoffel connection. In addition, the particle content of this theory can be seen by performing a linearized approximation to the field equations about a Minkowski background [2-4,41] (see Appendix A).

In order to perform the symplectic analysis, we will assume that the manifold $\mathcal{M}$ is topologically $\Sigma \times \Re$, where $\Sigma$ corresponds to a Cauchy surface without boundary $(\partial \Sigma=0)$ and $\Re$ represents an evolution parameter. Here, the $x^{\mu}$ are 
the coordinates that label the points of the three-dimensional manifold $\mathcal{M}$. In our notation, Greek letters run from 0 to 2 , while the lower case letters $(i, j, k, \ldots)$ run from 1 to 3 .
By using the symplectic variables, we find that the symplectic matrix $f_{i j}^{(0)}$ can be written as

$f_{i j}^{(0)}(x, y)=\left(\begin{array}{cccccc}2 \frac{\theta}{\mu} \epsilon^{a b} \eta_{i j} & 0 & -2 \theta \epsilon^{a b} \eta_{i j} & 0 & 0 & 0 \\ 0 & 0 & 0 & 0 & 0 & 0 \\ 2 \theta \epsilon^{a b} \eta_{i j} & 0 & 0 & 0 & -\epsilon^{a b} \eta_{i j} & 0 \\ 0 & 0 & 0 & 0 & 0 & 0 \\ 0 & 0 & \epsilon^{a b} \eta_{i j} & 0 & 0 & 0 \\ 0 & 0 & 0 & 0 & 0 & 0\end{array}\right) \delta^{2}(x-y)$.

By performing the $2+1$ decomposition of our fields without breaking the internal symmetry, we can write the action (1) as

$$
\begin{aligned}
S[A, e, \lambda]= & \int_{\Sigma}\left[\theta \epsilon^{a b}\left(2 e_{b i}+\frac{1}{\mu} A_{b i}\right) \dot{A}^{i}{ }_{a}+\epsilon^{a b} \lambda_{i b} \dot{e}^{i}{ }_{a}\right. \\
& +\epsilon^{a b} e^{i}{ }_{0}\left(\theta F_{a b i}+D_{a} \lambda_{b i}\right)+\frac{1}{2} \epsilon^{a b} \lambda^{i}{ }_{0} T_{a b i} \\
& \left.+\epsilon^{a b} A^{i}{ }_{0}\left(\theta T_{a b i}+\frac{1}{\mu} \theta F_{a b i}+f_{i j k} \lambda^{j}{ }_{a} e^{k}{ }_{b}\right)\right] d^{3} x,
\end{aligned}
$$

where $F_{a b}{ }^{i}=\partial_{a} A_{b}{ }^{i}-\partial_{b} A_{a}{ }^{i}+f^{i}{ }_{j k} A_{a}{ }^{j} A_{b}{ }^{k}, T_{a b}{ }^{i}=$ $D_{a} e_{b}{ }^{i}-D_{b} e_{a}{ }^{i}$, and the covariant derivative of $\lambda_{a}{ }^{i}$ is defined as $D_{a} \lambda_{b}{ }^{i}=\partial_{a} \lambda_{b}{ }^{i}+f^{i}{ }_{j k} A_{a}{ }^{j} \lambda_{b}{ }^{k}$. Here $a, b=1,2$ are space coordinate indices (the dot represents a derivative with respect to the evolution parameter). From (6) we can identify the following first-order Lagrangian density:

$$
\begin{aligned}
\mathcal{L}^{(0)}= & \theta \epsilon^{a b}\left(2 e_{b i}+\frac{1}{\mu} A_{b i}\right) \times \dot{A}^{i}{ }_{a}+\epsilon^{a b} \lambda_{i b} \dot{e}^{i}{ }_{a} \\
& +\epsilon^{a b} e^{i}{ }_{0}\left(\theta F_{a b i}+D_{a} \lambda_{b i}\right)+\frac{1}{2} \epsilon^{a b} \lambda^{i}{ }_{0} T_{a b i} \\
& +\epsilon^{a b} A^{i}{ }_{0}\left(\theta T_{a b i}+\frac{1}{\mu} \theta F_{a b i}+f_{i j k} \lambda^{j}{ }_{a} e^{k}{ }_{b}\right) .
\end{aligned}
$$

From the variational principle applied to the Lagrangian density (7), it is possible to write the symplectic equations of motion as

$f_{i j}^{(0)} \dot{\xi}^{(0) j}=\frac{\delta V^{(0)}(\xi)}{\delta \xi^{(0) i}}$,

where $f_{i j}^{(0)}=\frac{\delta}{\delta \xi(0) i} a_{j}^{(0)}(\xi)-\frac{\delta}{\delta \xi^{(0) j}} a_{i}^{(0)}(\xi)$, which is clearly antisymmetric, is known as the symplectic two-form, which yields the following symplectic variable set: $\xi^{(0) i}=\left(A_{a}^{i}\right.$, $\left.A^{i}{ }_{0}, e_{a}^{i}, e^{i}, \lambda^{i}{ }_{a}, \lambda^{i}{ }_{0}\right)$, the corresponding symplectic oneform $a^{(0)}{ }_{i}=\left(2 \theta \epsilon^{a b} e_{b i}+\frac{\theta}{\mu} \epsilon^{a b} A_{b i}, 0, \epsilon^{a b} \lambda_{b i}, 0,0,0\right)$, and the symplectic potential $V^{(0)}$ given by

$$
\begin{aligned}
V^{(0)}= & \epsilon^{a b} e^{i}{ }_{0}\left(\theta F_{a b i}+D_{a} \lambda_{b i}\right)+\frac{1}{2} \epsilon^{a b} \lambda^{i}{ }_{0} T_{a b i}+\epsilon^{a b} A^{i}{ }_{0} \\
& \times\left(\theta T_{a b i}+\frac{1}{\mu} \theta F_{a b i}+f_{i j k} \lambda^{j}{ }_{a} e^{k}{ }_{b}\right) .
\end{aligned}
$$

Clearly $f_{i j}^{(0)}$ is degenerate, which means that there are more degrees of freedom in the equations of motion (8) than physical degrees of freedom in the theory. We thus have a constrained theory, with constraints that must remove the unphysical degrees of freedom. The zero modes of this matrix turn out to be $\left(v_{1}^{(0)}\right)_{i}^{T}=\left(0, v^{A^{i} 0}, 0,0,0,0\right),\left(v_{2}^{(0)}\right)_{i}^{T}=$ $\left(0,0,0, v^{e^{i}}, 0,0\right)$, and $\left(v_{3}^{(0)}\right)_{i}^{T}=\left(0,0,0,0,0, v^{\lambda^{i}{ }_{0}}\right)$, where $v^{A^{i}{ }_{0}}, v^{e^{i}}$ and $v^{\lambda^{i}{ }_{0}}$ are arbitrary functions. By multiplying the two sides of (8) by these zero modes, we can obtain the following primary constraints:

$$
\begin{aligned}
\Xi_{i}^{(0)} & =\int \mathrm{d} x^{2}\left(v_{1}^{(0)}\right)_{j}^{T} \frac{\delta}{\delta \xi^{(0) j}} \int \mathrm{d} y^{2} V^{(0)} \\
& =\theta \epsilon^{a b} T_{a b i}+\frac{\theta}{\mu} \epsilon^{a b} F_{a b i}+\epsilon^{a b} f_{i j k} \lambda^{j}{ }_{a} e^{k}{ }_{b}=0, \\
\Theta_{i}^{(0)} & =\int \mathrm{d} x^{2}\left(v_{2}^{(0)}\right)_{j}^{T} \frac{\delta}{\delta \xi^{(0) j}} \int \mathrm{d} y^{2} V^{(0)} \\
& =\theta \epsilon^{a b} F_{a b i}+\epsilon^{a b} D_{a} \lambda_{b i}=0, \\
\Sigma_{i}^{(0)} & =\int \mathrm{d} x^{2}\left(v_{3}^{(0)}\right)_{j}^{T} \frac{\delta}{\delta \xi^{(0) j}} \int \mathrm{d} y^{2} V^{(0)}=\frac{1}{2} \epsilon^{a b} T_{a b i}=0 .
\end{aligned}
$$

Following the prescription of the symplectic formalism, we will analyze whether there are new constraints. To this aim, we impose a consistency condition on the constraints (11) as in the Dirac method:

$\dot{\Omega}^{(0)}=\frac{\delta \Omega^{(0)}}{\delta \xi^{(0) i}} \dot{\xi}^{(0) i}=0 \quad$ with

$\Omega^{(0)}=\Xi_{i}^{(0)}, \Theta_{i}^{(0)}, \Sigma_{i}^{(0)}$,

which means that these constraints must be preserved in time. The consistency condition on the primary constraints (12) and (8) can be rewritten as

$f_{k j}^{(1)} \dot{\xi}^{(0) j}=Z_{k}^{(1)}(\xi)$,

where

$f_{k j}^{(1)}=\left(\begin{array}{c}f_{i j}^{(0)} \\ \frac{\delta \Omega^{(0)}}{\delta \xi^{(0) j}}\end{array}\right) \quad$ and $\quad Z_{k}^{(1)}=\left(\begin{array}{c}\frac{\delta V^{(0)}}{\delta \xi^{(0) j}} \\ 0 \\ 0 \\ 0\end{array}\right)$. 
Thus the new symplectic matrix $f_{k j}^{(1)}$ is given by

$$
\begin{aligned}
& \left(\begin{array}{cccccr}
2 \frac{\theta}{\mu} \eta_{i j} & 0 & -2 \theta \eta_{i j} & 0 & 0 & 0 \\
0 & 0 & 0 & 0 & 0 & 0 \\
2 \theta \eta_{i j} & 0 & 0 & 0 & -\eta_{i j} & 0 \\
0 & 0 & 0 & 0 & 0 & 0 \\
0 & 0 & \eta_{i j} & 0 & 0 & 0 \\
0 & 0 & 0 & 0 & 0 & 0 \\
2 \frac{\theta}{\mu}\left(\eta_{i j} \partial_{a}-f_{i j k} A^{k}{ }_{a}-\mu f_{i j k} e^{k}{ }_{a}\right) & 0 & 2 \theta\left(\eta_{i j} \partial_{a}-f_{i j k} A^{k}{ }_{a}-\frac{1}{2 \theta} f_{i j k} \lambda^{k}{ }_{a}\right) & 0 & -f_{i j k} e^{k}{ }_{a} & 0 \\
2 \theta\left(\eta_{i j} \partial_{a}-f_{i j k} A^{k}{ }_{a}-\frac{1}{2 \theta} f_{i j k} \lambda^{k}{ }_{a}\right) & 0 & 0 & 0 & 0\left(\eta_{i j} \partial_{a}-f_{i j k} A_{a}^{k}\right) & 0 \\
-f_{i j k} e^{k}{ }_{a} & 0 & \left(\eta_{i j} \partial_{a}-f_{i j k} A_{a}^{k}\right) & 0 & 0 & 0
\end{array}\right) \\
& \times \epsilon^{a b} \delta^{2}(x-y) .
\end{aligned}
$$

Although $f_{k j}^{(1)}$ is not a square matrix, it still has the following linearly independent zero modes:

$$
\begin{aligned}
\left(v_{1}^{(1)}\right)^{j T}= & \left(\partial_{a} v^{j}-f^{j}{ }_{l m} A_{a}{ }_{a} v^{m}, v^{e^{j} 0},\right. \\
& \left.-f^{j}{ }_{l m} e^{l}{ }_{a} v^{m}, 0, f^{j}{ }_{l m} \lambda_{a}{ }^{l} v^{m}, 0, v^{j}, 0,0\right), \\
\left(v_{2}^{(1)}\right)^{j T}= & \left(-\frac{\mu}{2 \theta} f^{j}{ }_{l m} e^{l}{ }_{a} v^{m}, 0,0, v^{A^{j}{ }_{0}}, \partial_{a} v^{j}\right. \\
& \left.-f^{j}{ }_{l m} A^{l}{ }_{a} v^{m}-\mu f^{j}{ }_{l m} e^{l}{ }_{a} v^{m}, 0,0,0, v^{j}\right), \\
\left(v_{3}^{(1)}\right)^{j T}= & \left(-\frac{\mu}{2 \theta} f^{j}{ }_{l m} \lambda^{l}{ }_{a} v^{m}, 0, \partial_{a} v^{j}\right. \\
& \left.-f^{j}{ }_{l m} A^{l}{ }_{a} v^{m}, 0,-\mu f^{j}{ }_{l m} \lambda^{l}{ }_{a} v^{m}, v^{\lambda^{j}{ }_{0}}, 0, v^{j}, 0\right),
\end{aligned}
$$

where $v^{m}, v^{e_{0}^{j}}, v^{A_{0}^{j}}, v^{\lambda_{0}^{j}}$ are arbitrary functions. On the other hand, the matrix $Z_{k}^{(1)}$ is given by
This agrees completely with what was found in [43] by using the Dirac approach, however, in this formalism the constraints (19) arise as tertiary and quartic constraints, respectively. Furthermore, we can impose the consistency conditions on (19) to obtain the following system:

$f_{k j}^{(2)} \dot{\xi}^{(0) j}=Z_{k}^{(2)}(\xi)$,

where we have now

$f_{k j}^{(2)}=\left(\begin{array}{c}f_{i j}^{(1)} \\ \frac{\delta \Omega^{(1)}}{\delta \xi^{(0) j}}\end{array}\right), \quad \Omega^{(1)}=\Lambda, \Lambda_{0 a} \quad$ and

$Z_{k}^{(2)}=\left(\begin{array}{c}Z_{k}^{(1)} \\ 0 \\ 0\end{array}\right)$.

$$
\left(\begin{array}{c}
-2 \theta D_{a} e_{0 j}+f_{j l m} e_{0}{ }^{l} \lambda_{a}{ }^{m}+f_{j l m} \lambda_{0}{ }^{l} e_{a}{ }^{m}+2 \theta f_{j l m} A_{0}{ }^{l} e_{a}{ }^{m}-2 \frac{1}{\mu} \theta D_{a} A_{0 j} \\
0 \\
-D_{a} \lambda_{0 j}-2 \theta D_{a} A_{0 j}+f_{j l m} A^{l} \lambda_{0}{ }^{m}{ }_{a} \\
0 \\
-D_{a} e_{0 j}+f_{j l m} A_{0}^{l} e^{m}{ }_{a} \\
0 \\
0 \\
0 \\
0
\end{array}\right) \epsilon^{a b} \delta^{2}(x-y)
$$

By performing the contraction of the two sides of (13) with the zero modes (16), we can obtain the following constraints:

$\left.\left(v^{(1)}\right)_{k}^{T} Z_{k}^{(1)}\right|_{\Omega^{(0)}=0}=0$.

The substitution $\Omega^{(0)}=0$ guarantees that these constraints will drop from the remainder of the calculation. After a lengthy but straightforward calculation, from (18) we obtain the explicit form of the secondary constraints

$$
\Lambda=2 \epsilon^{a b} e_{a}^{i} \lambda_{i b}, \quad \Lambda_{0 a}=e^{i}{ }_{0} \lambda_{i a}-e^{i}{ }_{a} \lambda_{i 0} .
$$

It is easy to see that, even after calculating the symplectic matrix $f_{k j}^{(2)}$ and inserting the above constraints (19), the zero modes do not yield new constraints, which means that there are no further constraints in the theory, and thus our procedure comes to an end. We can now introduce the Lagrange multipliers for the constraints (11) and (19) into the Lagrangian density (7) in order to construct a new one,

$$
\mathcal{L}^{(3)}=\theta \epsilon^{a b}\left(2 e^{i}{ }_{b}+\frac{1}{\mu} A_{b i}\right) \dot{A}^{i}{ }_{a}
$$




$$
\begin{aligned}
& +\epsilon^{a b} \lambda_{i b} \dot{e}^{i}{ }_{a}-\epsilon^{a b}\left(\theta F_{a b i}+D_{a} \lambda_{b i}\right) \dot{\alpha}^{i} \\
& -\frac{1}{2} \epsilon^{a b} T_{a b i} \dot{\Gamma}^{i} \\
& -\epsilon^{a b}\left(\theta T_{a b i}+\frac{\theta}{\mu} F_{a b i}+f_{i j k} \lambda^{j}{ }_{a} e^{k}{ }_{b}\right) \dot{\beta}^{i}-\Lambda \dot{\varphi} \\
& -\Lambda_{0 a} \dot{\varphi}^{0 a},
\end{aligned}
$$

where the new symplectic potential $V^{(3)}$ vanishes since it is a linear combination of constraints reflecting the general covariance of the theory, namely, $V^{(3)}=\left.V^{(0)}\right|_{\Omega^{(0)}, \Omega^{(1)}=0}=$ 0 . On the other hand, the new Lagrange multipliers enforcing the constraints are $\dot{\alpha}^{i}=e_{0}^{i}, \dot{\beta}^{i}=A_{0}^{i}, \dot{\Gamma}^{i}=\lambda_{0}^{i}, \dot{\varphi}$, and $\dot{\varphi}^{0 a}$. Therefore, the new symplectic variable set is taken as

$\xi^{(3) i}=\left(A_{a}^{i}, \beta^{i}, e_{a}^{i}, \alpha^{i}, \lambda^{i}{ }_{a}, \Gamma^{i}, \varphi, \varphi^{0 a}\right)$.

Thus, the corresponding symplectic one-form is

$$
\begin{aligned}
a_{i}^{(3)}= & \left(\theta \epsilon^{a b}\left(2 e_{b i}+\frac{1}{\mu} A_{b i}\right),-\Xi^{(0)}, \epsilon^{a b} \lambda_{b i},\right. \\
& \left.-\Theta_{i}^{(0)}, 0,-\Sigma_{i}^{(0)},-\Lambda,-\Lambda_{0 a}\right) .
\end{aligned}
$$

By using these symplectic variables, an explicit calculation yields a singular symplectic matrix $f_{i j}^{(3)}=\frac{\delta}{\delta \xi^{(3) i}} a_{j}^{(3)}(\xi)-$ $\frac{\delta}{\delta \xi^{(3) j}} a_{i}^{(3)}(\xi)$. However, we have shown that there are no more constraints, therefore, the theory must have a local gauge symmetry. The zero modes of $f_{i j}^{(3)}$ turn out to be

$$
\begin{aligned}
\left(v_{1}^{(3)}\right)^{i T}= & \left(-\partial_{a} \zeta^{i}-f^{i}{ }_{j k} A_{a}{ }^{j} \zeta^{k}, \zeta^{i},-f^{i}{ }_{j k} e_{a}^{j} \zeta^{k}, 0,\right. \\
& \left.-f^{i}{ }_{j k} \lambda_{a}{ }^{j} \zeta^{k}, 0,0,0\right), \\
\left(v_{2}^{(3)}\right)^{i T}= & \left(-\frac{\mu}{2 \theta} f^{i}{ }_{j k} \lambda_{a}{ }^{j} \kappa^{k}, 0,-\partial_{a} \kappa^{i}\right. \\
& \left.-f^{i}{ }_{j k} A_{a}{ }^{j} \kappa^{k}, \kappa^{i},+\mu f^{i}{ }_{j k} \lambda_{a}{ }^{j} \kappa^{k}, 0,0,0\right), \\
\left(v_{3}^{(3)}\right)^{i T}= & \left(-\frac{\mu}{2 \theta} f^{i}{ }_{j k} e_{a}{ }^{j} \varsigma^{k}, 0,0,0,-\partial_{a} \varsigma^{i}\right. \\
& \left.-f^{i}{ }_{j k} A_{a}{ }^{j} \varsigma^{k}+\mu f^{i}{ }_{j k} e_{a}{ }^{j} \varsigma^{k}, \varsigma^{i}, 0,0\right) .
\end{aligned}
$$

\section{Gauge symmetry}

It is well known that the gauge symmetry determines the physical content of any gauge theory, therefore we need to know explicitly the fundamental gauge transformations of the theory. In agreement with the prescription of the symplectic formalism [24-26,33], the zero modes correspond to the generators of the gauge symmetry of the original theory (1) (see Appendix B), i.e.

$\delta_{G} \xi^{(3) i}=\left(v_{l}^{(3)}\right)^{i T} \epsilon^{l}$,

where $\left\{\left(v_{l}^{(3)}\right)^{i T}\right\}$ is the whole set of zero modes of singular symplectic matrix $f_{i j}^{(3)}$ and $\epsilon^{l}$ stand for arbitrary infinitesimal parameters. By using this fact, the generators (25) yield the following fundamental gauge transformations of the basic fields:

$$
\begin{aligned}
\delta_{G} A_{\alpha}{ }^{i}(x)= & -D_{\alpha} \zeta^{i}-\frac{\mu}{2 \theta} f^{i}{ }_{j k}\left(e_{\alpha}{ }^{j} \varsigma^{k}+\lambda_{\alpha}{ }^{j} \kappa^{k}\right), \\
\delta_{G} e_{\alpha}{ }^{i}(x)= & -D_{\alpha} \kappa^{i}-f^{i}{ }_{j k} e_{a}^{j} \zeta^{k} \\
\delta_{G} \lambda_{\alpha}{ }^{i}(x)= & -D_{\alpha} \varsigma^{i}-f^{i}{ }_{j k} \lambda_{\alpha}{ }^{j} \zeta^{k} \\
& +\mu f^{i}{ }_{j k}\left(\lambda_{a}{ }^{j} \kappa^{k}+e_{a}{ }^{j} \varsigma^{k}\right),
\end{aligned}
$$

where $\zeta^{i}, \kappa^{i}$, and $\zeta^{i}$ are the time-dependent gauge parameters. It is worth remarking that (27) correspond to the gauge symmetry of the theory, but not to diffeomorphisms. Nevertheless, it is known that an appropriate choice of the gauge parameters does, indeed, generate diffeomorphism (on-shell) $[44,47,49]$. Thus, we can redefine the gauge parameters as

$\zeta^{i}=-A^{i}{ }_{\mu} \varepsilon^{\mu}, \quad \kappa^{i}=-e^{i}{ }_{\mu} \varepsilon^{\mu}, \quad \varsigma^{i}=-\lambda^{i}{ }_{\mu} \varepsilon^{\mu}$,

where $\varepsilon^{\mu}$ is an arbitrary three-vector. In this manner from the fundamental gauge symmetry (27) and the mapping (28), we obtain

$$
\begin{aligned}
\delta_{G} e_{\alpha}{ }^{i}= & \mathfrak{L}_{\varepsilon} e_{\alpha}{ }^{i}-\varepsilon^{\mu} \epsilon_{\alpha \mu \nu}(\delta \lambda)^{\nu i}, \\
\delta_{G} A_{\alpha}{ }^{i}= & \mathfrak{L}_{\varepsilon} A_{\alpha}{ }^{i}+\mu \varepsilon^{\mu} \epsilon_{\alpha \mu \nu}\left[\frac{1}{2 \theta}(\delta A)^{v i}+(\delta \lambda)^{v i}\right], \\
\delta_{G} \lambda_{\alpha}{ }^{i}= & \mathfrak{L}_{\varepsilon} \lambda_{\alpha}{ }^{i} \\
& +2 \mu \theta \varepsilon^{\mu} \epsilon_{\alpha \mu \nu}\left[\frac{1}{2 \mu \theta}(\delta e)^{\nu i}-\frac{1}{2 \theta}(\delta A)^{v i}+(\delta \lambda)^{\nu i}\right],
\end{aligned}
$$

which are (on-shell) diffeomorphisms. On the other hand, diffeomorphism invariant theories have the Poincaré transformations, as off-shell symmetries, by construction [45,46]. Thus, in order to recover the Poincaré symmetries, we need to map the gauge parameters of the fundamental gauge symmetries ' $\delta_{G}$ ' (27) into those of the Poincaré symmetries. This is achieved through the field-dependent map between the gauge parameters (27) and the Poincaré ones [44]:

$\zeta^{i}=A^{i}{ }_{\mu} \varepsilon^{\mu}+\omega^{i}, \quad \kappa^{i}=e^{i}{ }_{\mu} \varepsilon^{\mu}, \quad \zeta^{i}=\lambda^{i}{ }_{\mu} \varepsilon^{\mu}$,

where $\varepsilon^{\mu}$ and $\omega^{i}$ are the parameters of translations and local Lorentz rotations, respectively, which together constitute the 6 gauge parameters of Poincaré symmetries in 3D. By using this map, it is seen that the gauge symmetries indeed reproduce the Poincaré symmetries, but modulo terms proportional to the equations of motion,

$$
\begin{aligned}
\delta_{G} e_{\alpha}{ }^{i}= & -\varepsilon^{\mu} \partial_{\mu} e_{\alpha}{ }^{i}-e_{\mu}{ }^{i} \partial_{\alpha} \varepsilon^{\mu}-f^{i}{ }_{j k} e_{\alpha}{ }^{j} \omega^{k} \\
& +\varepsilon^{\gamma} \epsilon_{\alpha \gamma \nu}(\delta \lambda)^{v i}, \\
\delta_{G} A_{\alpha}{ }^{i}= & -\partial_{\alpha} \omega^{i}-f^{i}{ }_{j k} A_{\alpha}{ }^{j} \omega^{k}-\varepsilon^{\mu} \partial_{\mu} A_{\alpha}{ }^{i}-A_{\mu}{ }^{i} \partial_{\alpha} \varepsilon^{\mu} \\
& -\mu \varepsilon^{\gamma} \epsilon_{\alpha \gamma \nu} \times\left[\frac{1}{2 \theta}(\delta A)^{\nu i}+(\delta \lambda)^{\nu i}\right], \\
\delta_{G} \lambda_{\alpha}{ }^{i}= & -\varepsilon^{\mu} \partial_{\mu} \lambda_{\alpha}{ }^{i}-\lambda_{\mu}{ }^{i} \partial_{\alpha} \varepsilon^{\mu}-f^{i}{ }_{j k} \lambda_{\alpha}{ }^{j} \omega^{k}
\end{aligned}
$$




$$
-2 \mu \theta \varepsilon^{\gamma} \epsilon_{\alpha \gamma \nu}\left[\frac{1}{2 \mu \theta}(\delta e)^{v i}-\frac{1}{2 \theta}(\delta A)^{v i}+(\delta \lambda)^{\nu i}\right],
$$

where the equations of motion $(\delta e)^{v i},(\delta A)^{v i}$, and $(\delta \lambda)^{v i}$ are defined in (2). We thus conclude that the Poincaré symmetry (31) as well as the diffeomorphisms (28) are contained in the fundamental gauge symmetry (27) only on-shell. In addition, the generators of such gauge transformations can be represented in terms of the zero modes, thereby making evident that the zero modes of the symplectic two-form encode all the information as regards the gauge structure of this theory.

\section{Faddeev-Jackiw brackets}

Finally, in order to invert the symplectic matrix and obtain the generalized Faddeev-Jackiw brackets and identify the physical degrees of freedom, we must introduce a gauge-fixing procedure, that is, new "gauge constraints". For convenience, we use the temporal gauge, namely, $A^{i}{ }_{0}=0, e^{i}{ }_{0}=0$, $\lambda^{i}{ }_{0}=0$, and $\varphi=$ cte (i.e. $\dot{\varphi}=0$ ). As a direct consequence, the term $\Lambda_{0 a}$ vanishes in the Lagrangian density. In this man- ner, we also introduce new Lagrange multipliers that enforce the gauge conditions, namely, $\rho_{i}, \omega_{i}, \tau_{i}$, and $\sigma$. Then the final one-form Lagrangian density reduces to

$$
\begin{aligned}
\mathcal{L}^{(4)}= & \theta \epsilon^{a b}\left(2 e_{b i}+\frac{1}{\mu} A_{b i}\right) \dot{A}_{a}^{i}+\epsilon^{a b} \lambda_{i b} \dot{e}_{a}^{i} \\
& -\left(\Xi_{i}^{(0)}-\rho_{i}\right) \dot{\beta}^{i}-\left(\Theta_{i}^{(0)}-\omega_{i}\right) \dot{\alpha}^{i} \\
& -\left(\Sigma_{i}^{(0)}-\tau_{i}\right) \dot{\Gamma}^{i}-(\Lambda-\sigma) \dot{\varphi} .
\end{aligned}
$$

Thus, we can identify the final symplectic variable set

$\xi^{(4) i}=\left(A_{a}^{i}, \beta^{i}, e_{a}^{i}, \alpha^{i}, \lambda^{i}{ }_{a}, \Gamma^{i}, \varphi, \rho_{i}, \omega_{i}, \tau_{i}, \sigma\right)$,

with the corresponding symplectic one-form

$$
\begin{aligned}
a_{i}^{(4)}= & \left(\theta \epsilon^{a b}\left(2 e_{b i}+\frac{1}{\mu} A_{b i}\right),\right. \\
& -\Xi_{i}^{(0)}+\rho_{i}, \epsilon^{a b} \lambda_{b i},-\Theta_{i}^{(0)}+\omega_{i}, 0, \\
& \left.-\Sigma_{i}^{(0)}+\tau_{i},-\Lambda+\sigma, 0,0,0,0\right) .
\end{aligned}
$$

After some algebra, we obtain the explicit form of the symplectic two-form $f_{i j}^{(4)}$

$\left.\begin{array}{cccccccccccc}\hline \frac{2 \theta}{\mu} F & -2 \frac{\theta}{\mu}(A+\mu C) & -2 \theta F & -2 \theta\left(A+\frac{D}{2 \theta}\right) & 0 & -C & 0 & 0 & 0 & 0 & 0 \\ 2 \frac{\theta}{\mu}(A+\mu C) & 0 & 2 \theta\left(A+\frac{D}{2 \theta}\right) & 0 & C & 0 & 0 & -\eta_{i j} & 0 & 0 & 0 \\ 2 \theta F & -2 \theta\left(A+\frac{D}{2 \theta}\right) & 0 & 0 & -F & -A & 2 I & 0 & 0 & 0 & 0 \\ 2 \theta\left(A+\frac{D}{2 \theta}\right) & 0 & 0 & 0 & -A & 0 & 0 & 0 & -\eta_{i j} & 0 & 0 \\ 0 & -C & F & A & 0 & 0 & -2 H & 0 & 0 & 0 & 0 \\ C & 0 & A & 0 & 0 & 0 & 0 & 0 & 0 & -\eta_{i j} & 0 \\ 0 & 0 & -2 I & 0 & 2 H & 0 & 0 & 0 & 0 & 0 & -1 \\ 0 & \eta_{i j} & 0 & 0 & 0 & 0 & 0 & 0 & 0 & 0 & 0 \\ 0 & 0 & 0 & \eta_{i j} & 0 & 0 & 0 & 0 & 0 & 0 & 0 \\ 0 & 0 & 0 & 0 & 0 & \eta_{i j} & 0 & 0 & 0 & 0 & 0 \\ 0 & 0 & 0 & 0 & 0 & 0 & 1 & 0 & 0 & 0 & 0\end{array}\right)$


$\left.\begin{array}{ccccccccccc}\frac{\mu}{2 \theta} \bar{F} & 0 & 0 & 0 & -\mu \bar{F} & 0 & 0 & -\bar{A} & -\frac{\mu}{2 \theta} \bar{D} & -\frac{\mu}{2 \theta} \bar{C} & 2 \mu e_{b}{ }^{j} \\ 0 & 0 & 0 & 0 & 0 & 0 & 0 & -\eta^{i}{ }_{j} & 0 & 0 & 0 \\ 0 & 0 & 0 & 0 & -\bar{F} & 0 & 0 & -\bar{C} \bar{C} & -\bar{A} & 0 & 2 e_{a}{ }^{l} \\ 0 & 0 & 0 & 0 & 0 & 0 & 0 & 0 & \eta_{j}^{i} & 0 & 0 \\ \mu \bar{F} & 0 & \bar{F} & 0 & 2 \theta \mu \bar{F} & 0 & 0 & \bar{D} & \mu \bar{D} & 2(\bar{A}-\mu \bar{C}) & -2 G \\ 0 & 0 & 0 & 0 & 0 & 0 & 0 & 0 & 0 & \eta^{i}{ }_{j} & 0 \\ 0 & 0 & 0 & 0 & 0 & 0 & 0 & 0 & 0 & 0 & 1 \\ \bar{A} & \eta_{j}^{i} & C \bar{F} & 0 & \bar{D} & 0 & 0 & 0 & 0 & 0 & 0 \\ \frac{\mu}{2 \theta} D & 0 & A & -\eta_{j}^{i} & \mu \bar{D} & 0 & 0 & 0 & \frac{\mu}{2 \theta} E & 0 & 0 \\ \frac{\mu}{2 \theta} \bar{C} & 0 & 0 & 0 & (2 \bar{A}-\mu \bar{C}) & -\eta^{i j} & 0 & 0 & 0 & \frac{\mu}{\theta} B & 2 \mu \bar{H} \\ -2 \mu e_{b}{ }^{i} & 0 & -2 e_{a}{ }^{l} & 0 & 2 G & 0 & 1 & 0 & 0 & -2 \mu \bar{H} & 0\end{array}\right) \delta^{2}(x-y)$,

where

$$
\begin{aligned}
& A=\epsilon^{a b}\left(\partial_{a} \eta_{i j}+f_{i k j} A_{a}{ }^{k}\right), \\
& C=\epsilon^{a b} f_{i k j} e_{a}{ }^{k}, D=\epsilon^{a b} f_{i k j} \lambda_{a}{ }^{k}, F=\epsilon^{a b} \eta_{i j}, \\
& H=\epsilon^{a b} e_{a j}, I=\epsilon^{a b} \lambda_{a j}, \\
& \bar{A}=\left(\partial_{a} \eta_{i j}+f_{i k j} A_{a}{ }^{k}\right), \\
& B=\epsilon^{a b} f_{i j k} f^{k}{ }_{l m} e_{a}{ }^{j} e_{b}{ }^{l}, \bar{C}=f_{i k j} e_{a}{ }^{k}, \\
& \bar{D}=f_{i k j} \lambda_{a}{ }^{k}, \quad E=\epsilon^{a b} f_{i j k} f^{k}{ }_{l m} \lambda_{a}{ }^{j} \lambda_{b}{ }^{m}, \\
& \bar{F}=\epsilon_{a b} \eta^{i j}, \quad G=2 \theta \mu e_{b}{ }^{l}+\lambda_{b}{ }^{l}, \quad \bar{H}=\epsilon^{a b} f_{i j k} e_{a}^{j} e_{b}^{k} .
\end{aligned}
$$

The generalized Faddeev-Jackiw bracket $\{,\}_{F-J}$ between two elements of the symplectic variable set (33), is defined as

$$
\left\{\xi_{i}^{(4)}(x), \xi_{j}^{(4)}(y)\right\}_{F-J} \equiv\left(f_{i j}^{(4)}\right)^{-1}
$$

We thus arrive at the non-vanishing Faddeev-Jackiw brackets for TMG,

$$
\begin{aligned}
& \left\{A^{i}{ }_{a}(x), A^{j}{ }_{b}(y)\right\}_{F-J}=\frac{\mu}{2 \theta} \eta^{i j} \delta^{2}(x-y), \\
& \left\{A^{i}{ }_{a}(x), \lambda^{j}{ }_{b}(y)\right\}_{F-J}=\mu \epsilon_{a b} \eta^{i j} \delta^{2}(x-y), \\
& \left\{\lambda^{i}{ }_{a}(x), \lambda^{j}{ }_{b}(y)\right\}_{F-J}=2 \theta \mu \epsilon_{a b} \eta^{i j} \delta^{2}(x-y), \\
& \left\{e^{i}{ }_{a}(x), \lambda^{j}{ }_{b}(y)\right\}_{F-J}=\epsilon_{a b} \eta^{i j} \delta^{2}(x-y) .
\end{aligned}
$$

These F-J brackets coincide with the Dirac brackets reported in [43]. In addition, we can carry out the counting of degrees of freedom as follows. There are 18 canonical variables $\left(e_{a}^{i}, \lambda_{a}^{i}, A^{i}{ }_{a}\right)$, and 17 independent constraints $\left(\Xi_{i}^{(0)}, \Theta_{i}^{(0)}, \Sigma_{i}^{(0)}, \Lambda, e^{i}{ }_{0}, A^{i}, \varphi\right)$. Thus, we conclude that 3D TMG has one physical degree of freedom (number of canonical variables - number of independent constraints), corresponding to the massive graviton, as expected.

\section{Summary and conclusions}

In this paper, the dynamical structure of TMG theory has been studied via the F-J framework. We have obtained the fundamental gauge structure as well as the physical content of this theory in an alternative way to that reported in $[42,43,47]$. It was shown that in the F-J approach is not necessary to classify the constraints into first- and second-class ones. In this respect, all the constraints are treated at the same footing. The correct identification of the constraints of TMG theory allowed us to show that there is one local physical degree of freedom, and obtain the gauge generators that yield the Poincaré symmetries and the diffeomorphisms by mapping the gauge parameters appropriately. Thereafter, the quantization brackets (F-J brackets) were obtained. Our results coincide with what has been previously obtained via the Dirac approach [43]. It is worth mentioning that there is no oneto-one correspondence between the constraints that we have obtained via the F-J method and those found via the Dirac formalism [43], though both approaches yield the same results. Our study suggests that the F-J method turns out to be more economical, unambiguous, and straightforward than Dirac's one. Finally, we would like to comment that according to our results the F-J approach could be useful for studying interesting features of models of massive gravity, which include TMG as a particular sector, for instance, topologically massive AdS gravity. This idea is in progress and will be the subject of forthcoming work [51].

Acknowledgments This work was supported by CONACyT under Grant No. CB-2014-01/240781. We would like to thank D. Grumiller for valuable comments and R. Cartas-Fuentevilla for a discussion of the subject and reading of the manuscript.

Open Access This article is distributed under the terms of the Creative Commons Attribution 4.0 International License (http://creativecomm ons.org/licenses/by/4.0/), which permits unrestricted use, distribution, and reproduction in any medium, provided you give appropriate credit to the original author(s) and the source, provide a link to the Creative Commons license, and indicate if changes were made.

Funded by SCOAP ${ }^{3}$. 


\section{Appendix A: Linearized analysis in metric formalism}

In this appendix, using the metric formulation of TMG given by the Eq. (5), we study the linearized theory as a perturbation of the metric about a Minkowski background solution, writing

$g_{\mu \nu}=\bar{g}_{\mu \nu}+h_{\mu \nu}$,

where $\bar{g}_{\mu \nu}$ is the Minkowski metric and $h_{\mu \nu}$ is the perturbation. To first-order in this perturbation, the Ricci tensor and the Ricci scalar are given by

$R_{\mu \nu}^{(1)}=\frac{1}{2}\left(-\bar{\nabla}^{2} h_{\mu \nu}-\bar{\nabla}_{\mu} \bar{\nabla}_{\nu} h+\bar{\nabla}^{\sigma} \bar{\nabla}_{\nu} h_{\sigma \mu}+\bar{\nabla}^{\sigma} \bar{\nabla}_{\mu} h_{\sigma \nu}\right)$,

$R^{(1)}=\bar{\nabla}_{\mu} \bar{\nabla}_{\nu} h^{\mu \nu}-\bar{\nabla}^{2} h$,

here $h \equiv \bar{g}^{\mu \nu} h_{\mu \nu}$, and $\bar{\nabla}$ is the covariant derivative constructed with the background metric. Using these expressions one can build the first-order correction of the Einstein, and Cotton tensors as

$G_{\mu \nu}^{(1)}=R_{\mu \nu}^{(1)}-\frac{1}{2} \bar{g}_{\mu \nu} R^{(1)}$,

$C_{\mu \nu}^{(1)}=\epsilon_{\mu}{ }^{\alpha \beta} \bar{\nabla}_{\alpha}\left(R_{\beta \nu}^{(1)}-\frac{1}{4} \bar{g}_{\beta \nu} R^{(1)}\right)$.

On the other hand, the linearized Bianchi identity becomes

$C_{\mu \nu}^{(1)}-C_{\nu \mu}^{(1)}=0$.

The last term in the right hand side of (A5) is totally antisymmetric on $\mu$ and $\nu$, and therefore merely subtracts the antisymmetric piece from the first term in the right hand side of (A5). We alternatively have

$C_{\mu \nu}^{(1)}=\frac{1}{2}\left(\epsilon_{\mu}{ }^{\alpha \beta} \bar{\nabla}_{\alpha} R_{\beta \nu}^{(1)}+\epsilon_{\nu}{ }^{\alpha \beta} \bar{\nabla}_{\alpha} R_{\beta \mu}^{(1)}\right)$.

Note also that it is not hard to verify that

$\bar{\nabla}^{\mu} C_{\mu \nu}^{(1)}=0, \quad$ and $\quad C_{\mu}^{(1) \mu}=0$.

Then the first-order correction of Eq. (5) is given by

$G_{\mu \nu}^{(1)}+\frac{1}{\mu} C_{\mu \nu}^{(1)}=0$.

Furthermore from the trace of this equation one finds that: $R^{(1)}=0$, independent of $\mu$. Substituting this back, we therefore find that Eq. (A9) can be written as

$G_{\mu \nu}^{(1)}+\frac{1}{\mu} \epsilon_{\mu}^{\alpha \beta} \bar{\nabla}_{\alpha} G_{\beta \nu}^{(1)}=0$.

Now we consider the transverse (divergenceless) and traceless conditions on the Minkowski background as

$\bar{\nabla}^{\mu} h_{\mu \nu}=0, \quad$ and $\quad h_{\mu}^{\mu}=0$.
By making use of these conditions (A11), Eq. (A10) may be recast into the following form:

$\bar{\nabla}^{2}\left(\delta_{\mu}^{\beta}+\frac{1}{\mu} \epsilon_{\mu}^{\alpha \beta} \bar{\nabla}_{\alpha}\right) h_{\beta \nu}$.

Furthermore, this equation can be expressed compactly as

$$
\left[\mathcal{O}(0)^{2} \mathcal{O}(\mu) h\right]_{\mu \nu}=0 \text {, }
$$

by introducing two mutually commuting operators as

$$
\mathcal{O}(0)_{\mu}^{\beta} \equiv \epsilon_{\mu}^{\alpha \beta} \bar{\nabla}_{\alpha}, \quad \text { and } \quad \mathcal{O}(\mu)_{\mu}^{\beta} \equiv \delta_{\mu}^{\beta}+\frac{1}{\mu} \epsilon_{\mu}^{\alpha \beta} \bar{\nabla}_{\alpha} .
$$

Since the two operators conmute, Eq. (A13) has two branches of solutions. First, we have the massive graviton $h_{\mu \nu}^{M}$, given by

$$
\left[\mathcal{O}(\mu) h^{M}\right]_{\mu \nu}=h_{\mu \nu}^{M}+\frac{1}{\mu} \epsilon_{\mu}^{\alpha \beta} \bar{\nabla}_{\alpha} h_{\beta \nu}^{M}=0 .
$$

The other branch is the massless graviton $\breve{h}_{\mu \nu}$, given by

$$
[\mathcal{O}(0) \breve{h}]_{\mu \nu}=\epsilon_{\mu}^{\alpha \beta} \bar{\nabla}_{\alpha} \breve{h}_{\beta \nu}=0,
$$

which is also solution of Einstein gravity: $G_{\mu \nu}=0$. Now, let us define the linear operator $\mathcal{O}(-\mu)_{\mu}^{\beta} \equiv \delta_{\mu}^{\beta}-\frac{1}{\mu} \epsilon_{\mu}{ }^{\alpha \beta} \bar{\nabla}_{\alpha}$, which conmutes with $\mathcal{O}(\mu)$. By acting on (A15) with $\mathcal{O}(-\mu)$, we get the second-order equation for the massive graviton,

$\left[\bar{\nabla}^{2}-\mu^{2}\right] h_{\mu \nu}^{M}=0$.

Similarly, in the massless case, the second-order equation is given by

$$
\bar{\nabla}^{2} \breve{h}_{\mu \nu}=0 \text {. }
$$

Then the mass of the massive graviton can be identified by comparing the second-order equation of motion of the massive graviton with that of the massless graviton, therefore, the mass of the massive graviton is $m=\sqrt{\mu^{2}}$. In addition, Eq. (A15) propagates a single mode, which has spin-2, because $h$ is a symmetric traceless second-order tensor, therefore, Eq. (A17) is exactly the Fierz-Pauli equation describing a massive spin-2 field in Minkowski spacetime.

\section{Appendix B: Gauge symmetry}

We will assume that all the FJ constraints have been identified and therefore only the zero modes associated with gauge symmetries are still present. In this manner, the final symplectic Lagrangian can be written as

$$
\begin{aligned}
L(\xi)= & a_{i}(\xi) \dot{\xi}^{i}+\dot{\gamma}_{a} \Omega^{a}-V(\xi) \quad(i=1,2,3, \ldots, N), \\
& \times(a, b=1,2, \ldots, M),
\end{aligned}
$$


here the $\Omega^{a}$ are the complete set of FJ constraints and either the $\xi$ or the $\gamma$ form a set of gauge fields. Now, the symplectic matrix, namely $\bar{f}_{k l}$, constructed with the $\xi^{i}$ variables, is not singular, hence $\operatorname{det} \bar{f}_{k l} \neq 0$. Now let us call $f$ the symplectic matrix constructed out by using the $\xi$ and the $\gamma$; that symplectic matrix is singular and will be given by

$f=\left(\begin{array}{cc}\bar{f} & \frac{\partial \Omega}{\partial \xi} \\ -\left(\frac{\partial \Omega}{\partial \xi}\right)^{T} & 0\end{array}\right)$,

hence (B2) may have M zero modes of the form

$v_{k}^{a}=\left(\begin{array}{c}-(\bar{f})^{-1} \frac{\partial \Omega}{\partial \xi} \\ 1^{a}\end{array}\right)$

where $\left(1^{a}\right)$ is a $(M \times 1)$ column of zeros except its $a$ th entry [26]. Now, let us assume that the gradient of the potential is orthogonal to all zero modes, hence, they must be the generators of the symmetry transformation that leave the action invariant. In this manner, the symmetry of the action over the constraint surface is given by

$\delta \xi_{i}=-(\bar{f})^{-1} \frac{\partial \Omega}{\partial \xi} \epsilon_{l}$,
$\delta \gamma_{a}=-\epsilon_{I}$.

Here the $\epsilon_{l}$ form a set of infinitesimal parameters that characterize the transformations. It is important to comment that these transformations may reflect either the gauge or reparametrization properties of an invariant theory [26]. In fact, the first relation of the transformations (B4) is equivalent to one of the Dirac gauge transformations obtained from the first-class constraints. The latter does not have an easy description in the canonical formalism, this can be seen in models such as the Floreanini and Jackiw chiral boson and 2D Maxwell fields [52].

Furthermore, let us finish the appendix showing the invariance of the action. In fact, it is well known that the symmetries are defined by those variations $\delta \xi$ such that the functional variation of the action vanishes,

$$
\begin{aligned}
\delta S & =\int \mathrm{d} t\left(\frac{\partial L}{\partial \xi_{k}}-\partial_{t} \frac{\partial L}{\partial \dot{\xi}_{k}}\right) \delta \xi_{k} \\
& \equiv \int \mathrm{d} t\left(f_{k m} \dot{\xi}^{m}-\frac{\partial V}{\partial \xi_{k}}\right) \delta \xi_{k}=0 .
\end{aligned}
$$

Therefore, this expression defines the gauge symmetry; if there exists some variation $\delta \xi_{k}$ satisfying Eq. (B5), then the transformation

$\xi_{k} \longrightarrow \xi_{k}+\delta \xi_{k}$

is a symmetry of the action S. Hence, we can construct a variation $\delta \xi_{k}$ satisfying (B5) on the constraints surface, given as

$\delta \xi_{k}=\left(v_{l}\right)_{k} \epsilon^{l}$.
Therefore, since $\left(v^{l}\right)_{i}$ are the zero modes on the constraints surface, they must satisfy the equation of motion, i.e.

$$
\begin{aligned}
& \int \mathrm{d} t\left(f_{k m} \dot{\xi}_{m}-\frac{\partial V}{\partial \xi_{k}}\right)\left(v^{l}\right)_{k} \epsilon_{l} \\
& =\int \mathrm{d} t \epsilon_{l}\left(v^{l}\right)_{k}^{T}\left(f_{k m} \dot{\xi}_{m}-\frac{\partial V}{\partial \xi_{k}}\right)=\epsilon_{l} \Omega^{(l)} .
\end{aligned}
$$

And this shows that the action is invariant under displacements in directions orthogonal to the gradient of the potential.

\section{References}

1. S. Deser, R. Jackiw, S. Templeton, Three-dimensional massive gauge theories. Phys. Rev. Lett. 48, 975 (1982)

2. S. Deser, R. Jackiw, S. Templeton, Ann. Phys. 140, 372 (1982)

3. S. Deser, R. Jackiw, S. Templeton, Ann. Phys. 185, 406 (1988)

4. S. Deser, R. Jackiw, S. Templeton, Ann. Phys. 281, 409 (2000)

5. E.A. Bergshoeff, O. Hohm, P.K. Townsend, Massive gravity in three dimensions. Phys. Rev. Lett. 102, 201301 (2009)

6. V.A. Rubakov, P.G. Tinyakov, Infrared-modified gravities and massive gravitons. Phys. Usp. 51, 759 (2008)

7. K. Hinterbichler, Theoretical aspects of massive gravity. Rev. Mod. Phys. 84, 671 (2012)

8. C. de Rhan, Massive gravity. Living Rev. Rel 17, 7 (2014)

9. H.R. Afshar, E.A. Bergshoeff, W. Merbis, Interacting spin-2 fields in three dimensions. JHEP 01, 040 (2015)

10. S. del Pino, G. Giribet, A. Toloza, J. Zanelli, From Lorentz-ChernSimons to massive gravity in 2+1 dimensions. JHEP 06, 113 (2015)

11. O. Miskovic, R. Olea, Background-independent charges in topologically massive gravity. JHEP 12, 046 (2009)

12. C. Deffayet, J. Mourad, G. Zahariade, Covariant constraints in ghost free massive gravity. JCAP 01, 032 (2013)

13. M. Fierz, W. Pauli, On relativistic wave equation for paticles of arbitrary spin in an electromagnetic field. Proc. R. Soc. Lond. A. 173, 211 (1939)

14. D. Boulware, S. Deser, Can gravity have a finite range? Phys. Rev. D. 6, 3368 (1972)

15. P. Carmelli, A. Nicolis, M. Papucci, E. Trincherine, Ghost massive gravity. JHEP 0509, 003 (2005)

16. C. Rovelli, Partial observables. Phys. Rev. D. 65, 124013 (2002)

17. R. Banerjee, S. Gangopadhyay, P. Mukherjee and D. Roy, Symmetries of topological gravity with torsion in the hamiltonian and lagrangian formalisms, JHEP 02 (2010)

18. P.A.M. Dirac, Lectures Notes on Quantum Mechanics (Yeshiva University, New York, 1964)

19. L.D. Faddeev, R. Jackiw, Hamiltonian reduction of unconstrained and constrained systems. Phys. Rev. Lett. 60, 1692 (1988)

20. L. Castellani, Symmetries in the contrained Hamiltonian system. Ann. Phys. 143, 357 (1982)

21. T. Hanson, A. Egge, C. Teitelboim, Constraints Hamiltonian Systems (Accademia Nazionale dei Lincei, Roma, 1978)

22. M. Henneaux, C. Teitelboim, Quantization of Gauge Systems, Princeton (Princeton University Press, New Jersey, 1991)

23. J. Barcelos-Neto et al., Symplectic quantization of constrained systems. Mod. Phys. Lett. A. 7, 1737 (1992)

24. J. Barcelos-Neto, C. Wotzasek, Faddeev-Jackiw quantization and constraints. Int. J. Mod. Phys. A. 7, 4981 (1992)

25. H. Montani, R. Montemayor, Lagrangian approach to a symplectic formalism for singular systems. Phys. Rev. D. 58, 125018 (1998) 
26. H. Montani, C. Wotzasek, Faddeev-Jackiw quantization of nonabelian systems. Mod. Phys. Lett. A. 8, 3387 (1993)

27. J. Antonio, Garcia, Josep M. Pons Equivalence of Faddeev-Jackiw and Dirac approaches for gauge theories. Int. J. Mod. Phys. A. 12, 451 (1997)

28. L. Liao, Y.C. Huang, Non-equivalence of Faddeev-Jackiw method and Dirac-Bergmann algorithm and the modification of FaddeevJackiw method for keeping the equivalence. Ann. Phys. 322, 2469 (2007)

29. R. Bufalo, B.M. Pimentel, Higher-derivative non-Abelian gauge fields via the Faddeev-Jackiw formalism. Eur. Phys. J. C 74, 2993 (2014)

30. E.M.C. Abreu, A.C.R. Mendes, C. Neves, W. Oliveira, R.C.N. Silva, QCD gauge symmetries through Faddeev-Jackiw symplectic method. JHEP. 06, 098 (2013)

31. Z.-W. Long, J. Jing, Faddeev-Jackiw approach to the noncommutativity. Phys. Lett. B. 560, 128 (2003)

32. E.M.C. Abreu, A.C.R. Mendes, C. Neves, W. Oliveira, R.C.N. Silva, C. Wotzasek, Obtaining non-Abelian field theories via the Faddeev-Jackiw symplectic formalism. Phys. Lett. A. 375, 3603 (2010)

33. C. Wotzasek, Faddeev-Jackiw approach to hidden symmetries. Ann. Phys. 243, 73 (1995)

34. D.S. Kulshreshtha, H.J.W. Muller-Kirsten, Faddeev-Jackiw quantization of selfdual fields. Phys. Rev. D. 45, 393 (1992)

35. Y.-C. Huang, J.-L. Yang, Modified Faddeev-Jackiw quantization of massive non-Abellian Yang-Mills fields and Lagrange multiplier fields. Phys. Lett. B. 668, 438 (2008)

36. S. Koh, S. Shin, Hamiltonian analysis of linearized extension of Horava-Lifshitz gravity. Phys. Lett. B. 696, 426 (2011)

37. A. Escalante, J. Manuel-Cabrera, Faddeev-Jackiw quantization of an Abelian and non-Abelian exotic action for gravity in three dimensions. Ann. Phys. 361, 585 (2015)

38. A. Escalante, M. Zárate, Dirac and Faddeev-Jackiw quantization of a five-dimensional Stüeckelberg theory with a compact dimension. Ann. Phys. 353, 163 (2014)
39. A. Escalante, O. Rodríguez-Tzompantzi, Dirac's and generalized Faddeev-Jackiw brackets for Einstein's theory in the $G \rightarrow 0$ limit. Ann. Phys. 364, 136 (2016)

40. D. Grumiller, R. Jackiw, N. Johansson, Canonical analysis of cosmological topologically massive gravity at the chiral point, MITCTP 3957, UUITP-12/08, (2008). arXiv:0806.4185

41. W. Li, W. Song, A. Strominger, Chiral gravity in three dimensions. JHEP 0804, 082 (2008)

42. M.-I. Park, Constraint dynamics and gravitons in three dimensions. JHEP 0809, 084 (2008)

43. M. Blagojevic, B. Cvetkovic, Canonical structure of topologically massive gravity with a cosmological constant. JHEP 05, 073 (2009)

44. M. Blagojevic, Gravitation and Gauge Symmetries (IOP, Bristol, 2002)

45. T.W.B. Kibble, Lorentz invariance and the gravitational field. J. Math. Phys. 2, 212 (1961)

46. R. Utiyama, Invariant theoretical interpretation of interaction. Phys. Rev. 101, 1597 (1956)

47. S. Carlip, Constraint algebra of topologically massive AdS gravity. JHEP 0810, 078 (2008)

48. A. Escalante, L. Carbajal, Hamiltonian study for Chern-Simons and Pontryagin theories. Ann. Phys. 326, 323-339 (2011)

49. A. Escalante, O. Rodríguez Tzompantzi, Hamiltonian dynamics and gauge symmetry for three-dimensional Palatini theory with cosmological constant, JHEP 05, 073 (2014)

50. A. Escalante, I. Rubalcava-Garcia, A pure Dirac's canonnical analysis for four-dimensional BF theories. Int. J. Geom. Methods Mod. Phys. 09, 1250053 (2012)

51. A. Escalante, O. Rodríguez-Tzompantzi (work in preparation)

52. R. Floreanini, R. Jackiw, Self-dual fields as charge-density solitons. Phys. Rev. Lett. 59, 1873 (1987) 